\title{
On wireless channel modelling : Extraction of channel from channel measurements
}

\author{
Aastha Gupta , Thushara D. Abhayapala and Tony S. Pollock \\ Department of Information Engineering \\ Research School of Information Sciences and Engineering \\ Australian National University \\ Canberra, ACT-0200 \\ Australia \\ Email:aastha.gupta@rsise.anu.edu.au, thushara.abhayapala@anu.edu.au
}

\begin{abstract}
Next generation systems are using directional antennas for indoor high data rate devices. This arises a need for measurement and modelling of directional channels. Indoor environments also introduce extreme multipath behaviour into the directional channel. Directional measurements raise the problem of complicated data sets with 2-D spatio-temporal information. In order to analyse these complex data sets, it is first imperative to make sure the data set actually represents the propagation channel and is not influenced by the measuring apparatus. In order to construct the propagation channel, we discuss a novel technique of eliminating such effects from the measured data set. The method involves solving sets of simultaneous equations in a three stage process. Firstly, the data is cleaned in the spatial domain, secondly in the in the time domain and finally the channel parameters are extracted. The technique is verified on real and simulation data sets.
\end{abstract}

\section{INTRODUCTION}

The next generation in indoor communications is arguably the Wireless Personal Area Networks (WPANs). WPANs propose millimeter-wave frequencies and allow for fast, high data rate wireless communication between devices in an indoor environment. They enable the complete transformation of any indoor office/home environment to wireless. The most commercially lucrative advantage of mm-wave WPANs is the extremely high data rates that can potentially be attained, typically exceeding 2 GB per second [1]. High gain directional antennas are proposed in order to cater for the required high data rates. In addition to providing high data rates, directional antennas are also useful to encounter high attenuation conditions. High attenuation in millimeter-wave propagation, is overcome by focussing the signal power between the highly directional antennas. The combination of millimeter-wave using high gain directional antennas is an appropriate solution for high data rate indoor propagation.

In order to design and build WPAN devices it is needed to first analyse and model the wireless propagation channel. Multiple reflections and scattering in an indoor environment, cause the propagation of the signal to occur in a multipath manner [2], [3], [4], [5], [6] . Angle of Arrival (AOA) measurements are an essential part of modelling any multipath channel. AOA measurements are performed by rotating a directional receiver antenna while keeping the transmitter antenna fixed. Such measurements provide both spatial and temporal information of the channel simultaneously.

The measurements that are obtained from the measuring equipment represent the Radio channel. These measurements include the effects of the measuring apparatus into the propagation channel. In order to study the propagation channel it is essential to remove the contaminating effects of the measuring apparatus from the measurement data. Radio channel measurements using directional antennas are also contaminated by the antenna gain pattern and the signal processing filter characteristics. Antennas have a significant effect on the measurement results. They alter the multipath signals arriving from different directions, by weighting them with the directional antenna gain pattern. This distorts the arriving signal and the impulse response of the channel. As the antenna pattern and its effect is direction dependent, its deconvolution with the channel impulse response is a highly challenging task, it requires a separate computation for each multipath signal arriving from a different direction. This can only be applied if the measurements allow the directions of the multipaths to be determined [7].

In literature, most measurements do not perform a deconvolution at all, they simply present results combined for the channel and antenna [8]. Some researchers perform a deconvolution; however ignore the directional dependence of the antenna's impulse response providing results averaged over all directions [8]. Without the removal of the antenna pattern the measured channel response is inaccurate and provides a statistical concatenation of error in the subsequent channel model.

Some other researchers have used image resolution tools such as CLEAN and SAGE to remove deteriorating effects from the measurement data [8], [9], [10], [11], [12], [13], [14]. Such techniques require a point spread function (PSF) of the channel data image [15]. A data image is a 3-D view of the data amplitudes as shown by Fig. 1. The PSF requires apriori information about the propagation channel which is not available and has to be approximated. The approximation of the PSF is a vital input to these tools and even a slight inaccuracy in the PSF alters the result enormously. Therefore, the use of these tools is risky and deteriorating the data set 


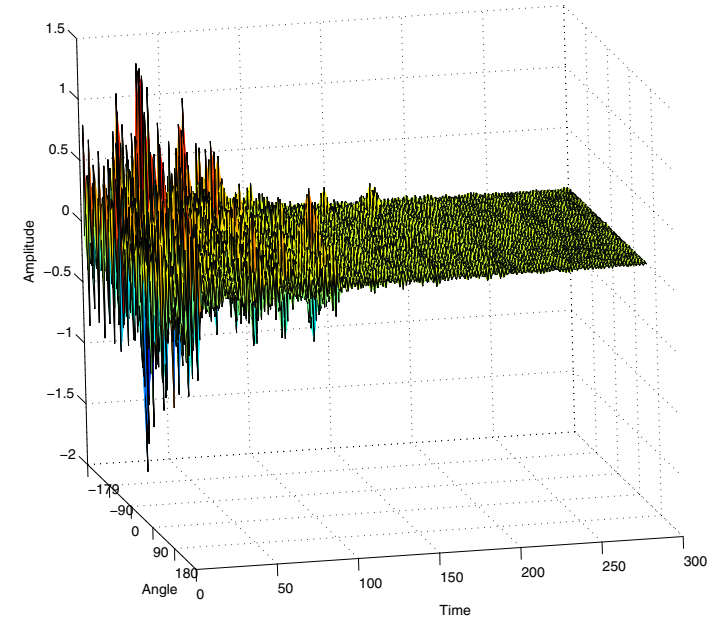

Fig. 1. A plot of raw measurement data. The data was collected by pointing a directional receiver antenna at different directions in indoor room while keeping the omnidirectional transmitter antenna fixed.

further is more likely than actually improving them.

Thus, there arises the need for a more fundamental approach to process the measurement data, which removes effects of the measuring equipment without the need for any apriori information of the propagation channel. In order to remove the spatial and temporal distortion effects, a step-by step novel technique is devised in this paper.

\section{RADIO CHANNEL MODEL}

We assume that the channel measurements are performed by transmitting a pulse signal $p(t)$ using an omnidirectional transmit antenna and a directional receiver antenna pointing to different directions for each measurement. Let the directional response (antenna pattern) of the receiver antenna be $b\left(\phi-\theta_{n}\right)$, where $\theta_{n}$ is the look direction (direction of interest) and $\phi \in$ $[0,2 \pi)$. Then the output signal for the look direction $\theta_{n}$, as shown by Fig. 8 is

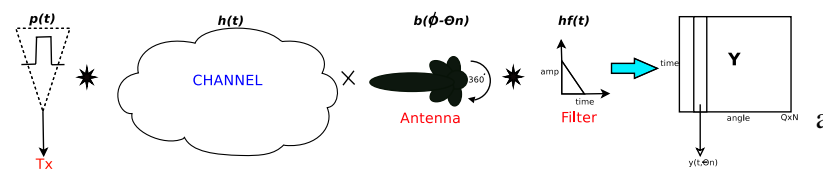

Fig. 2. A pictorial view of signal propagation.

$$
y\left(t, \theta_{n}\right)=\int_{\phi} p(t) * h(t, \phi) b\left(\phi-\theta_{n}\right) d \phi * h_{f}(t)+v(t)
$$

where $h(t, \phi)$ is the channel impulse response, $p(t)$ is the transmitted pulse, $h_{f}(t)$ represents the linear time invariant filter characteristics of the receiver apparatus and $v(t)$ represents noise in the channel measurements. Note that $p(t)$ and $h_{f}(t)$ are independent of the angle $\phi$ over which the integration is performed. Thus,

$$
y\left(t, \theta_{n}\right)=f(t) * \int_{\phi} h(\phi, t) b\left(\phi-\theta_{n}\right) d \phi+v(t),
$$

where

$$
f(t)=p(t) * h_{f}(t)
$$

We can approximate the integration by a summation to obtain:

$$
y\left(t, \theta_{n}\right)=f(t) * \sum_{m=0}^{M} h\left(\phi_{m}, t\right) b\left(\phi_{m}-\theta_{n}\right) \Delta \phi,
$$

where $\Delta \phi$ is the sampling interval of the antenna pattern $b(\phi)$, and $M$ is the number of antenna samples.

In AOA channel measurements, it is customary to make a number of independent measurements at different look directions, so as to estimate the channel more completely. By writing (4) for $n$ look directions, $n=1, \ldots, N$; we get the following matrix equation:

$$
\mathbf{y}(t)=\mathbf{B h}(t) * f(t)+\mathbf{v}(t)
$$

where $N$ is the number of look directions over which the antenna is rotated,

$$
\mathbf{B}=\left[\begin{array}{ccc}
b\left(\phi_{1}-\theta_{1}\right) & \ldots & b\left(\phi_{M}-\theta_{1}\right) \\
\vdots & \vdots & \vdots \\
b\left(\phi_{1}-\theta_{N}\right) & \ldots & b\left(\phi_{M}-\theta_{N}\right)
\end{array}\right]_{N \times M} \Delta \phi
$$

is the antenna sampling matrix,

$$
\begin{gathered}
\mathbf{h}(t)=\left[\begin{array}{c}
h\left(t, \phi_{1}\right) \\
h\left(t, \phi_{2}\right) \\
\vdots \\
h\left(t, \phi_{M}\right)
\end{array}\right]_{M \times 1}, \\
\mathbf{v}(t)=\left[\begin{array}{c}
v\left(t, \theta_{1}\right) \\
v\left(t, \theta_{2}\right) \\
\vdots \\
v\left(t, \theta_{N}\right)
\end{array}\right]_{N \times 1}, \\
\mathbf{y}(t)=\left[\begin{array}{c}
y\left(t, \theta_{1}\right) \\
y\left(t, \theta_{2}\right) \\
\vdots \\
y\left(t, \theta_{N}\right)
\end{array}\right]_{N \times 1} .
\end{gathered}
$$

For simplicity reasons, $\mathbf{v}(t)$ will be omitted from the rest of this paper. By including noise, we could further study the effect of different noise levels on the proposed model. However, it is out of the scope of the current paper and will be investigated in future journal publications. 


\section{ANTENNA PATTERN ELIMINATION}

In (5), the effect of the receiver antenna pattern is only present in $\mathbf{B}$. Thus, we can preprocess the received signal vector $\mathbf{y}(t)$ to eliminate the effect of the antenna gain pattern. This could be achieved in a least squared sense by multiplying (5) with the pseudo inverse of $\mathbf{B}$ :

$$
\hat{\mathbf{y}}(t)=\mathbf{B}^{\dagger} \mathbf{y}(t)=\mathbf{h}(t) * f(t)
$$

where $\hat{\mathbf{y}}(t)=\left[\hat{y}\left(t, \phi_{1}\right) \hat{y}\left(t, \phi_{2}\right) \ldots \hat{y}\left(t, \phi_{M}\right)\right]$ and

$$
\mathbf{B}^{\dagger}=\left(\mathbf{B}^{T} \mathbf{B}\right)^{-1} \mathbf{B}^{T} .
$$

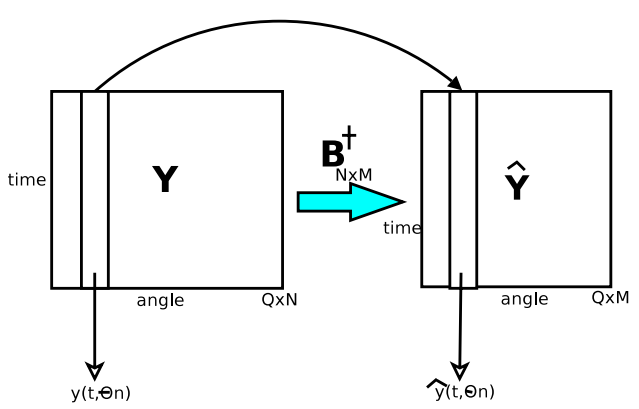

Fig. 3. Antenna elimination.

In this way, we can remove the antenna pattern from the measured received signal. The resulting antenna removed signal is represented by $\hat{\mathbf{y}}(t)$ for $m=1, \ldots, M$. The values of the antenna removed signal are given by the elements of $\hat{\mathbf{y}}(t)$ for $M$ different angles. A summary of the above process is depicted in fig: 6 .

\section{Temporal Cleaning - Filter Elimination}

In channel measurement campaigns, the transmitted pulse is generated using a sum of sinusoids. However, the exact shape of the transmitted pulse is usually not known.

Generally, from channel measurement data it can be seen that there is delay developed due to the electronic equipment. Such delays are often observed when dealing with robust electronic components that do not have an instantaneous response time to the received signal. Again, just for illustration purposes, a linear decay triangular filter can be used to mimic the electronic system. Also, because of multipath propagation, several delayed copies of the transmitted pulse are received. When these multipath copies pass through the delay filter, there is a resulting cumulative distortion effect. Therefore, the effect of the filter becomes significant in channel modelling and should be removed. In this section, we show how to remove the combined effect of non-ideal pulse shape $p(t)$ and filtering associated with the measurement equipment $h_{f}(t)$.

Consider the signal received from an angle $\phi_{m}$ over all time samples. Let this signal be represented by $\tilde{y}\left(t, \phi_{m}\right)$ where $\mathrm{m}$ is a constant for a particular calculation and the time $t_{q}$ of the samples is for $q=1, \ldots, Q$. Note that $\tilde{y}\left(t, \phi_{m}\right)$ is different from $\hat{y}\left(t, \theta_{m}\right)$ in Section III, as depicted from Figures 3 and 4 . The $\hat{y}\left(t, \theta_{m}\right)$ represents the antenna cleaned signal for a particular direction $\theta_{m}$, whereas $\tilde{y}\left(t, \phi_{m}\right)$ represents the antenna cleaned signal for a particular time $t$. So,

$$
\tilde{y}\left(t, \phi_{m}\right)=h\left(t, \phi_{m}\right) * f(t),
$$

where, $f(t)$ is given by

$$
f(t)=p(t) * h_{f}(t) .
$$

Now, consider the special case when $\phi_{m}=0^{\circ}$, i.e when the antenna is focussed in the Line-Of-Sight (LOS) direction with the transmitter antenna. In this case, we assume no multipath propagation and hence, $h(t, 0) \approx \delta(t)$. Then (12) reduces to $\tilde{y}(t, 0)=f(t)$.

Therefore, the unknown $f(t)$ is given by the LOS measured signal and we do not need to know the actual pulse or filter to determine the propagation channel. Suppose, we have preprocessed the data to remove the antenna effects using the method in Section III, and so now, we need to remove the effect of both the unknown pulse and receiver filter. Hence, the task is to remove effects of the combined filter and pulse, i.e $f(t)$ from the preprocessed data $\widehat{\mathbf{Y}}$.

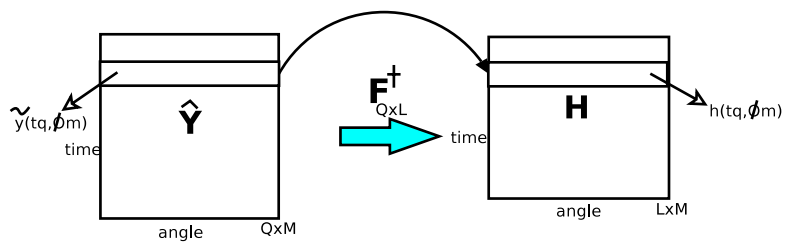

Fig. 4. Filter Elimination.

Now consider the time signals $\tilde{y}\left(t, \phi_{m}\right)$ for all cases of $m$ and write the convolution in (12) as

$$
\tilde{y}\left(t, \phi_{m}\right)=\int_{0}^{T_{c}} f(t-\tau) h\left(\tau, \phi_{m}\right) d \tau,
$$

where $T_{c}$ is the duration of $f(t)$. Let us approximate the integration by a summation to get

$$
\tilde{y}\left(t, \phi_{m}\right) \cong \sum_{s=0}^{S} f\left(t-\tau_{s}\right) h\left(\tau_{s}, \phi_{m}\right) \Delta \tau,
$$

where, $\Delta \tau$ is a suitable sampling time. For all time samples $t_{q}, q=1, \ldots, Q$ and fixed angle direction $m$, we can write a system of simultaneous equations to form a matrix equation

$$
\tilde{\mathbf{y}}\left(\phi_{m}\right)=\mathbf{F h}\left(\phi_{m}\right),
$$

where,

$$
\mathbf{F}=\left[\begin{array}{ccc}
f\left(t_{1}-\tau_{1}\right) & \ldots & f\left(t_{1}-\tau_{S}\right) \\
\vdots & \vdots & \vdots \\
f\left(t_{Q}-\tau_{1}\right) & \ldots & f\left(t_{Q}-\tau_{S}\right)
\end{array}\right]_{Q \times S} \Delta \tau
$$




$$
\tilde{\mathbf{y}}\left(\phi_{m}\right)=\left[\begin{array}{c}
\tilde{y}\left(t_{1}, \phi_{m}\right) \\
\tilde{y}\left(t_{2}, \phi_{m}\right) \\
\vdots \\
\tilde{y}\left(t_{Q}, \phi_{m}\right)
\end{array}\right]_{Q \times 1},
$$

and

$$
\mathbf{h}\left(\phi_{m}\right)=\left[\begin{array}{c}
h\left(\tau_{1}, \phi_{m}\right) \\
h\left(\tau_{2}, \phi_{m}\right) \\
\vdots \\
h\left(\tau_{S}, \phi_{m}\right)
\end{array}\right]_{S \times 1} .
$$

We then multiply (16) by the pseudo-inverse of $\mathbf{F}$, denoted by $\mathbf{F}^{\dagger}\left(\mathbf{F}^{\dagger}=\left(\mathbf{F}^{T} \mathbf{F}\right)^{-1} \mathbf{F}^{T}\right)$ to get

$$
\mathbf{h}\left(\phi_{m}\right)=\mathbf{F}^{\dagger} \tilde{\mathbf{y}}\left(\phi_{m}\right) \text {. }
$$

As a result, not only do we eliminate the effect of the $f(t)$, but we also estimate the channel $\left(\mathbf{h}\left(\phi_{m}\right)\right)$ for each direction $\phi_{m}$. It has been obtained after the elimination of the systems' filter function on the preprocessed data $\tilde{y}\left(t, \phi_{m}\right)$ as shown by fig:7. As a result of this theoretical analysis, we eliminate both the antenna and filter effects from the measurement data, and consecutively obtain the propagation channel data $\mathbf{H}$. The above analyses can be summarised by fig: 3 .
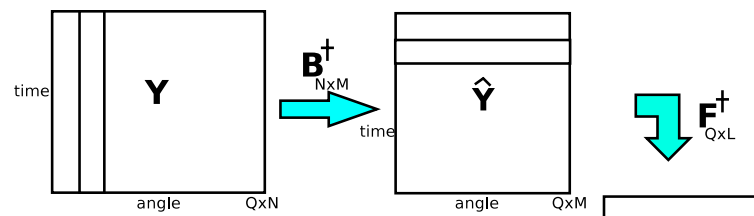

Fig. 5. Channel determination.

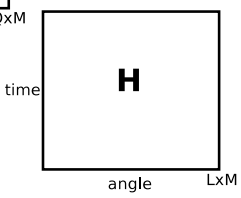

\section{Applications}

We have used the technique described in this paper to derive the $60 \mathrm{GHz}$ indoor channel model based on measurements provided by NICT Japan. measured and measured 2 show plots of preprocessed and post-processed measurement data for a single direction. It is clear from these figures that the post-processed data has more well-defined and uncorrelated multipath components than the preprocessed data. Detailed results have been reported in [16] and cannot be provided in this paper due to confidentiality of information.

The Theoretical analysis presented in Sections II, III and IV, was also applied to artificially created data sets. The analysis was verified when the channel matrix $\mathbf{H}$ obtained after the analysis perfectly matched the artificially generated $\mathbf{H}$. The simulation was performed for a number of different channels with taps of variable delay, amplitude and AOAs. Indeed it was found that the theoretical channel matrix $\mathbf{H}$ obtained after extraction is similar to the one simulated. As a result, it can

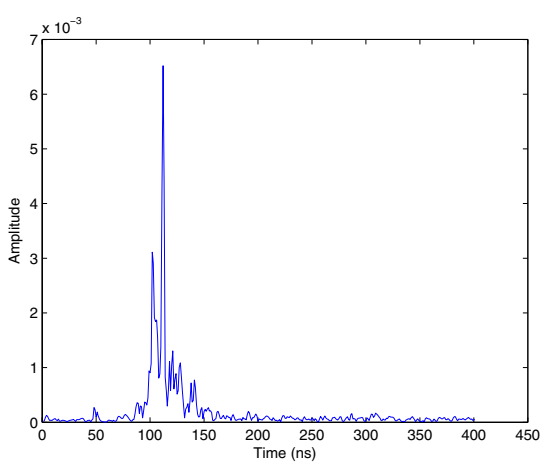

Fig. 6. Unprocessed data for $\theta_{m}=180^{\circ}$.

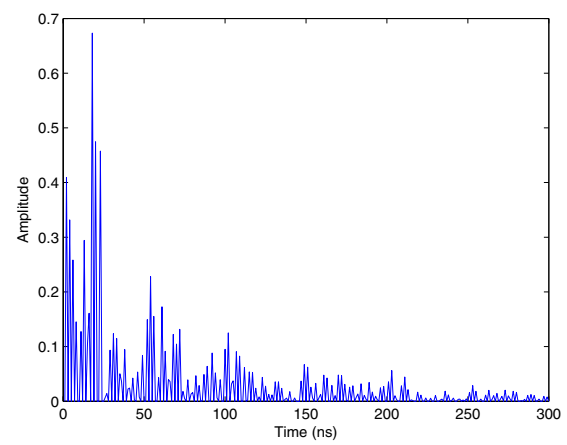

Fig. 7. Processed data for $\theta_{m}=180^{\circ}$.

be shown that we have successfully devised a procedure that extracts the true propagation channel from the measured radio channel.

\section{CONCLUSion}

In order to obtain an accurate modelling of the propagation environment, it is essential to first eliminate any contaminating effects from the measurement data. In this paper a theoretical technique has been devised that removes distortions of the measuring antenna and signal processing filter from any directional measurement data set. This method does not require any apriori information of the propagation channel and so is reliable to use. The method has been successfully used on real directional measurement data sets. Simulations have also been performed to further verify the technique.

\section{ACKNOWLEDGMENT}

The Authors would like to thank NICT, Japan for providing this channel modelling group with appropriate measurements.

\section{REFERENCES}

[1] NICTA, "802.15 WPAN alternative physical Layer System Requirements Document," IEEE standards Association, IEEE Standard No.802.15-050353-07-003c, 2005. 
[2] R. Vaughan and J.B. Andersen, Channel, Propagation and Antennas for Mobile Communications, The Institution of Electrical Engineers, 2003.

[3] M. Fiacco, M. Parks, H. Radi and S.R. Saunders, "Final Report - Indoor Propagation Factors at $17 \mathrm{GHz}$ and $60 \mathrm{GHz}$," University of Surrey, UK, Study on behalf of the UK Radio Communications Agency, August, 1998.

[4] M. Flament, "On $60 \mathrm{GHz}$ Wireless Communication Systems," Chalmers University of Technology, Gothenburg, Sweden, Thesis for the Degree of Licentiate of Engineering, no. 365L, 2000.

[5] J. Medbo and P. Schramm, "Channel Models for HIPERLAN/2 in Different Indoor Scenarios," ETSI EP BRAN, vol. 3ERI085B, 30th March, 1998.

[6] J. Hubner, S. Zeisberg, K. Koora, J. Borowski and A. Finger, "Simple Channel Model for $60 \mathrm{GHz}$ Indoor Wireless LAN Design Based on Complex Wideband Measurements," Proc. of 47th IEEE Veh. Tech. Conf., vol. 2, pp. 1004-1008, May, 1997.

[7] A.F. Molisch, "Ultrawideband propagation channels - theory, measurement and modelling," in Proc. IEEE Veh. Tech. Conf., 2005.

[8] Q.H. Spencer, B.D. Jeffs, M.A. Jensen and M. Rice, "A Statistical Model for Angle of Arrival in Indoor Multipath Propagation,Proc. IEEE Veh. Tech. Conf., vol. 3, pp. 1415-1419, May 1997.

[9] C.C. Chong, C.M. Tan, D.I. Laurenson, S. McLaughlin, M.A. Beach and A.R. Nix, "Joint Detection-Estimation of Directional Channel Parameters using the 2-D Frequency Domain SAGE Algorithm with Serial Intereference Cancellation," IEEE Intl. Conf. on Communications, vol. 2, pp. 906-910, May 2002.

[10] J.M. Cramer, R.A. Scholtz and M.Z. Win, "Evaluation of an UltraWideband Propagation Channel," TRW Space \& Electronics, IEEE Standard No.802.15-02/368r5-SG3a.

[11] B.H. Fleury, D. Dahlhaus, R. Heddergott and M. Tschudin, "Wideband Angle of Arrival Estimation Using the SAGE Algorithm," IEEE Intl. Symp. on Spread Spectrum Techniques and Applications Proc., vol. 1, pp. 79-85, Sep. 1996.

[12] K.I. Pedersen, B.H. Fleury and P.E. Mogensen, "High Resolution of Electromagnetic Waves in Time-Varying Radio Channels," IEEE Intl. Symp. on Personal Indoor and Mobile Radio Comm., vol. 2, pp. 650654, Sep. 1997.

[13] B.H. Fleury, M. Tschudin, R. Heddergott, D. Dahlhaus and K.I. Pedersen, "Space-Alternating Generalised Expectation-Maximisation Algorithm," IEEE Journal on Selected Areas in Communications, vol. SAC-17, 3, pp. 434-450, 1999.

[14] J.A. Fessler and A.O. Hero, "Evaluation of an Ultra-Wideband Propagation Channel," IEEE Trans. on Signal Processing, vol. 42, 10, pp. 2664-2677, October, 1994.

[15] D.L. Fried, "Analysis of the CLEAN Algorithm and implications for superresolution," Optical Society Am. A. vol. 12, 5, pp. 853-860, May, 1995.

[16] Z. Krusevac, S. Krusevac, A. Gupta, T. Pollock, "NICT Indoor 60 $\mathrm{GHz}$ Channel Measurements and Analysis update," IEEE standards Association, 802.15-06-0113-01-003c, 2006. 